\title{
Research Article \\ Correlations in Output and Overflow Traffic Processes in Simple Queues
}

\author{
Don McNickle \\ Received 11 April 2007; Accepted 8 August 2007 \\ Recommended by Paul Cowpertwait
}

We consider some simple Markov and Erlang queues with limited storage space. Although the departure processes from some such systems are known to be Poisson, they actually consist of the superposition of two complex correlated processes, the overflow process and the output process. We measure the cross-correlation between the counting processes for these two processes. It turns out that this can be positive, negative, or even zero (without implying independence). The models suggest some general principles on how big these correlations are, and when they are important. This may suggest when renewal or moment approximations to similar processes will be successful, and when they will not.

Copyright ( 2007 Don McNickle. This is an open access article distributed under the Creative Commons Attribution License, which permits unrestricted use, distribution, and reproduction in any medium, provided the original work is properly cited.

\section{Introduction}

We consider a finite capacity queueing system, usually of the M/G/C/L class. Customers who arrive when there are already $L+C$ customers in the system overflow. We can identify three traffic processes in the customers leaving the system: the overflow process formed by customers who find the system full, the output process formed by customers who complete service, and these two processes, superposed together, from the departure process.

It is well known that in a number of cases in this class of queues, the departure process, when considered as an isolated process, is Poisson. For example:

(i) in the limit, the departure process from an $\mathrm{M} / \mathrm{M} / \mathrm{C} / \mathrm{L}$ queue is Poisson (Boes [1]);

(ii) interdeparture times from an $\mathrm{M} / \mathrm{G} / \mathrm{C} / 0$ queue are exponentially distributed and are independent in the limit (Shanbag and Tambouratzis [2]).

Yet the complex behaviour present in these processes cannot be determined by examining the departure process in isolation. If we take the simplest case, of outputs and overflows 
from an $\mathrm{M} / \mathrm{M} / 1 / 0$ queue, then although both the output and the overflow process are renewal, neither is Poisson. The overflow process has a hyperexponential distribution and the output process is of phase type with two phases. Yet when superposed, they produce a Poisson process. Now the superposition of two independent renewal processes is Poisson if and only if both are Poisson processes. So the conclusion must be that the overflow and output processes cannot be independent. In this paper, we investigate the degree of this dependence by considering the cross-correlation functions of the two processes. We show that these cross-correlations can be positive, negative, or even uniformly zero, depending on the parameters of the process. Only in the simplest case do they have a clear form. However the models do suggest some general principles for the signs and sizes of these correlations, and when they are important. There have been a number of methods suggested for moment- or renewal-type approximations to these processes or similar ones, for example, the equivalent random method from classical telephone theory (Cooper [3]). (See also Whitt [4], Albin [5], Albin and Kai [6], and Johnson [7]). Having some qualitative knowledge about correlations should enhance the reliability and the appropriate use of these methods. There are a large number of papers on queues with similar correlated arrivals; see, for example, Adan and Kulkarni [8], and Heindl [9] (and other papers in the special issue of Performance Evaluation).

Individually, the characteristics of the overflow and output traffic processes are usually quite easy to determine. The output process is of Markov renewal type, (see Disney and Kiessler [10, Theorem 3.3, page 172]). This collapses to a renewal output process if and only if either (i) the service times are all zero with probability 1 , (ii) $L=0$, (iii) $L=1$ with the service times being constant, or (iv) $L=\infty$ with the service times being exponential (see Disney and Kiessler [10, Theorem 3.5] for the proof of this).

Where the departure process has a Markov renewal representation, the overflow process is renewal, since it consists of the times between entries to a particular state in the Markov renewal process. Hence the distribution of times between overflows can be established by the usual filtering arguments.

\section{Methodology}

2.1. Cross-correlations between the overflow and the output processes. The measures that we will use for the dependence between the overflow and output processes are the crosscovariance and cross-correlation of the processes that count the number of outputs, $N^{o}(t)$, and overflows, $N^{o v}(t)$, in $(0, t]$. Thus we define

$$
\begin{gathered}
c \operatorname{cov}\left(N^{o}(t) N^{o v}(t)\right)=E\left[N^{o}(t) N^{o v}(t)\right]-E\left[N^{o}(t)\right] E\left[N^{o v}(t)\right], \\
c \operatorname{cor}\left(N^{o}(t) N^{o v}(t)\right)=\frac{c \operatorname{cov}\left(N^{o}(t) N^{o v}(t)\right)}{\sqrt{\operatorname{Var}\left(N^{o}(t)\right) \operatorname{Var}\left(N^{o v}(t)\right)}} .
\end{gathered}
$$

Since we need the joint distribution of $N^{o}(t)$ and $N^{o v}(t)$, we consider systems where the entire departure process can be represented by an $n$-state Markov renewal process $(X, T)$ with semi-Markov kernel $Q^{d}(t)$. Usually the state will be the number of customers left behind by a departure. Entries to one subclass of states (often the $n$th state) represent overflows, while entries to the remaining states are outputs. 
The Laplace-Stieltjes transform of the Markov renewal kernel is

$$
R_{s}=\left(I-Q_{s}^{d}\right)^{-1}
$$

where $Q_{s}^{d}$ is the Laplace-Stieltjes transform of $Q^{d}(t)$. Çinlar [11, page 165] gives a general expression for the expectation of the product of the numbers of visits by time $t, N_{j}(t)$ and $N_{k}(t)$, to states $j$ and $k$, for a general finite-state Markov renewal process. That is, conditional on the process starting in state $i$ :

$$
E_{i}\left[N_{j}(t) N_{k}(t)\right]=\delta_{j k} R_{i j}^{\prime}(t)+R_{i j}^{\prime} * R_{j k}^{\prime}(t)+R_{i k} * R_{k j}^{\prime}(t)
$$

The expected product of the numbers of visits to states $j$ and $k$ in steady state is

$$
\sum_{i=1}^{n} \alpha_{i} E_{i}\left[N_{j}(t) N_{k}(t)\right] .
$$

Here $*$ stands for the convolution operation, $R_{i j}^{\prime}(t)=R_{i j}(t)-\delta_{i j}$, to ensure that the state occupied at time zero is not counted in the expected number of entries; $n$ is the number of states, and $\boldsymbol{\alpha}=\left[\alpha_{1} \cdots \alpha_{n}\right]$ is the steady state vector for the Markov chain imbedded in the Markov renewal process.

2.2. The calculation method and verification steps. The calculations were carried out using the symbolic algebra package Maple to do the matrix operations and invert the resulting Laplace-Stieltjes transforms. Since these programmes are reasonably complex (up to 100 lines of Maple), a number of checks to verify the calculation were carried out. For example, in each of the cases where the marginal departure process is Poisson with rate $\lambda$, it was verified that $\boldsymbol{\alpha}$ and $Q_{s}^{d}$ satisfy (e is a column vector of ones)

$$
\alpha Q_{s}^{d} e=\frac{\lambda}{\lambda+s}
$$

The matrix of expected products of the numbers of visits to states $j$ and $k$ in steady state was verified to be symmetric in $j$ and $k$ (this is a particularly good check since it is produced at the end of a long sequence of distinctly asymmetric calculations), and finally the simulation study (see Section 4) was also run on a number of the cases that could be solved analytically. It produced entirely consistent results.

\section{Results}

3.1. $\mathbf{M} / \mathbf{M} / \mathbf{1} / \mathbf{0}$. We start with the simplest case of no storage with all distributions being exponential. While this is a very simple model, it is also the simplest case of the Erlang-B formula from classical telephone theory. We let the state of the system be the number of customers left behind by a departure. Thus each entry to state 0 is an output and entries to state 1 are overflows. With $\lambda$ being the rate of the arrival Poisson process, and $\mu$ being the service rate, the Laplace-Stieltjes transform of the semi-Markov kernel of the departure 
4 Journal of Applied Mathematics and Decision Sciences

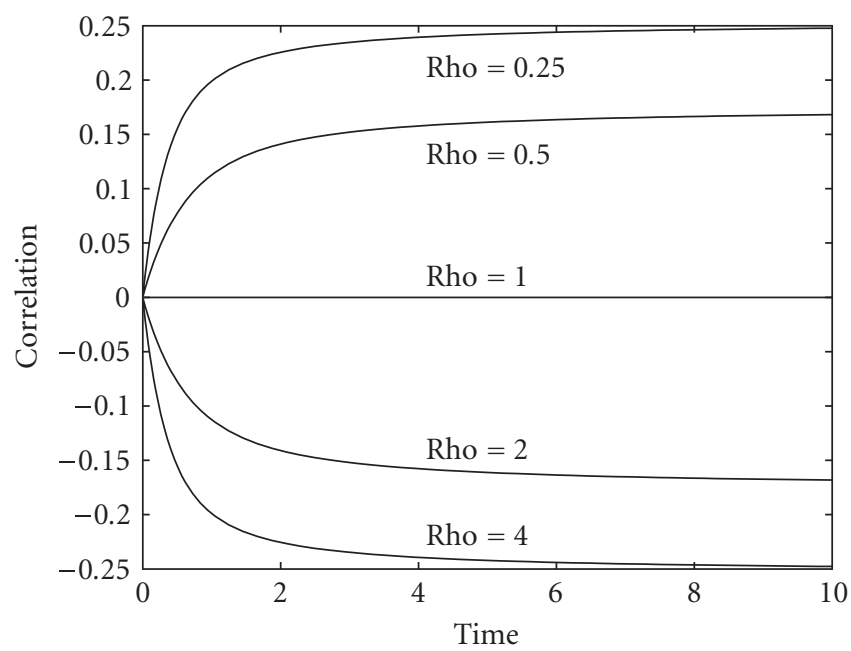

Figure 3.1. M/M/1/0 output and overflow cross-correlations.

process (see also Disney and Kiessler [10, page 84$]$ ) is

$$
Q_{s}^{d}=\left(\begin{array}{cc}
\frac{\lambda \mu}{(\lambda+s)(\lambda+\mu+s)} & \frac{\lambda^{2}}{(\lambda+s)(\lambda+\mu+s)} \\
\frac{\mu}{(\lambda+\mu+s)} & \frac{\lambda}{(\lambda+\mu+s)}
\end{array}\right)
$$

The Laplace-Stieltjes transform of the Markov renewal kernel is

$$
R_{s}=\left(I-Q_{s}^{d}\right)^{-1}=\left(\begin{array}{cc}
\frac{(\lambda+s)(\mu+s)}{s(\lambda+\mu+s)} & \frac{\lambda^{2}}{s(\lambda+\mu+s)} \\
\frac{\mu(\lambda+s)}{s(\lambda+\mu+s)} & \frac{\lambda^{2}+2 \lambda s+\mu s+s^{2}}{s(\lambda+\mu+s)}
\end{array}\right)
$$

Applying (2.3) and (2.4) to appropriate terms from (3.2) leads (with the assistance of Maple) to the following expression for the cross-covariance of the number of outputs and the number of overflows by time $t$ (starting from steady state):

$$
c \operatorname{cov}\left(N^{o}(t) N^{o v}(t)\right)=\frac{\mu \lambda^{2}\left(\lambda-\mu-\left(\lambda^{2}-\mu^{2}\right) t-(\lambda-\mu) e^{-(\lambda+\mu) t}\right)}{(\lambda+\mu)^{4}} .
$$

Thus we immediately see that when $\lambda=\mu$, the cross-covariance is zero for all times. Of course by Disney and Kiessler [10, Theorem 3.5], this cannot imply that the overflow and output processes are independent. So this is one of those peculiar situations where zero correlations do not imply independence. The variances of the numbers of outputs and of 
overflows are

$$
\begin{gathered}
\operatorname{Var}\left(N^{o}(t)\right)=\frac{\mu \lambda\left(\left(\lambda^{3}+\mu^{3}+\mu^{2} \lambda+\mu \lambda^{2}\right) t+2 \lambda \mu\left(1-e^{-(\lambda+\mu) t}\right)\right)}{(\lambda+\mu)^{4}}, \\
\operatorname{Var}\left(N^{o v}(t)\right)=\frac{\lambda^{2}\left(\left(\lambda^{3}+\mu^{3}+5 \mu^{2} \lambda+5 \mu \lambda^{2}\right) t-2 \lambda \mu\left(1-e^{-(\lambda+\mu) t}\right)\right)}{(\lambda+\mu)^{4}} .
\end{gathered}
$$

The cross-correlation of the number of outputs and the number of overflows by time $t$ can then be calculated from (3.3) and (3.4). The cross-correlation can be seen to be identical, except for a change of sign, when the values of $\lambda$ and $\mu$ are interchanged. Thus if $\lambda<\mu$, the number of outputs and the number of overflows are positively correlated over any time interval, while if $\lambda>\mu$ they are negatively correlated. To demonstrate this symmetry, the cross-correlations are plotted in Figure 3.1 for values of $(\lambda, \mu)=\{(1,4),(1,2),(1,1),(2,1)$, $(4,1)\}$ (traffic intensities of Rho $=0.25,0.5,1,2,4)$.

So the cross-correlations reduce monotonically with increasing traffic intensity, and increase monotonically in absolute value with time. Taking the limit of the crosscorrelation expression gives

$$
\lim _{t \rightarrow \infty} c \operatorname{cor}\left(N^{o}(t) N^{o v}(t)\right)=\frac{\lambda \mu(\mu-\lambda)}{\sqrt{\left(\lambda^{2}+4 \lambda \mu+\mu^{2}\right)\left(\lambda^{3} \mu+\lambda \mu^{3}\right)}} .
$$

This limiting expression was also found by a simple direct method in Disney and Kiessler.

3.2. Systems with storage, $M / M / 1 / 1, M / M / 1 / 2$. We now add one or two units of storage to the system. Again an appropriate state for the departure Markov renewal process is the number of customers left behind by a departure. We give only the results for $M / M / 1 / 2$. Those for $\mathrm{M} / \mathrm{M} / 1 / 1$ are similar, but less pronounced. Labelling the states as $\{0,1,2,3\}$, each entry to state 0,1 , or 2 is an output and entries to state 3 are overflows. The LaplaceStieltjes transform of the semi-Markov kernel of the departure process is

$$
Q_{s}^{d}=\left(\begin{array}{cccc}
\frac{\lambda \mu}{(\lambda+s)(\lambda+\mu+s)} & \frac{\lambda^{2} \mu}{(\lambda+s)(\lambda+\mu+s)^{2}} & \frac{\lambda^{3} \mu}{(\lambda+s)(\lambda+\mu+s)^{3}} & \frac{\lambda^{4}}{(\lambda+s)(\lambda+\mu+s)^{3}} \\
\frac{\mu}{\lambda+\mu+s} & \frac{\lambda \mu}{(\lambda+\mu+s)^{2}} & \frac{\lambda^{2} \mu}{(\lambda+\mu+s)^{3}} & \frac{\lambda^{3}}{(\lambda+\mu+s)^{3}} \\
0 & \frac{\mu}{\lambda+\mu+s} & \frac{\lambda \mu}{(\lambda+\mu+s)^{2}} & \frac{\lambda^{2}}{(\lambda+\mu+s)^{2}} \\
0 & 0 & \frac{\mu}{(\lambda+\mu+s)} & \frac{\lambda}{(\lambda+\mu+s)}
\end{array}\right) .
$$

The Markov renewal matrix does not now have an informative form, but the method is as before. Since the results are no longer symmetric in $\lambda$ and $\mu$, we take, as we will from now on, the mean service time to be 1 . Plots of the cross-correlations, for traffic intensities of $0.5,0.8,1$, and 2 are given in Figure 3.2. The smallest cross-correlations occur at a traffic intensity of about 0.8 . So the symmetry and monotone nature of the $M / M / 1 / 0$ results have gone (possibly due to the fact that the output process is no longer renewal), but 


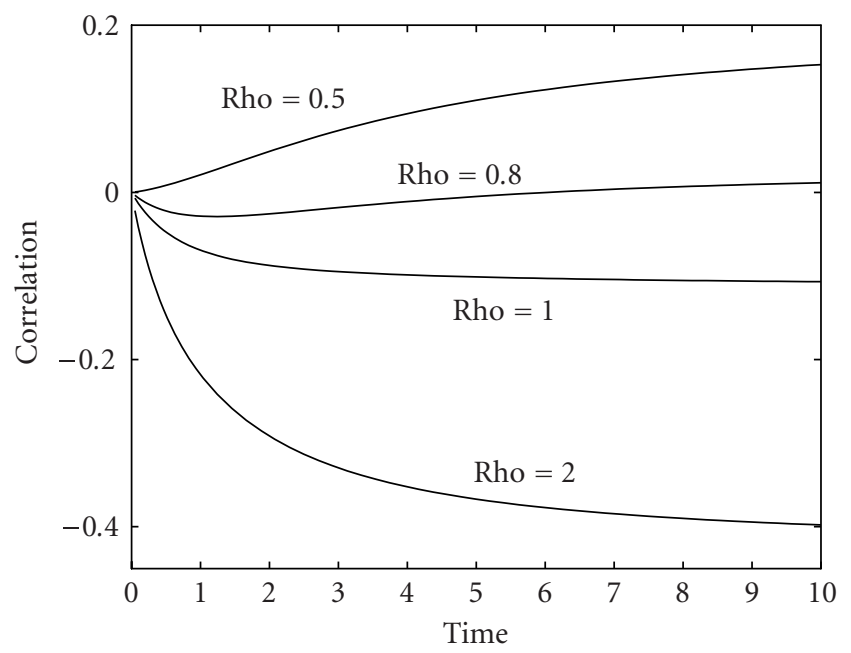

Figure 3.2. M/M/1/2 output and overflow cross-correlations.

the general principles of positive cross-correlations at low traffic intensities and negative cross-correlations at high traffic intensities, which will be discussed further in Section 3.5, are starting to emerge.

3.3. $\mathbf{M} / \mathbf{M} / 2 / 0$. With two servers and no storage we assume that when both servers are idle, an arrival selects a server by tossing a coin (the results when the servers are tested in a fixed order are very similar). We take the state of the process to be $\{i, j, k\}$, where $i=1$ or 2 is the server from which the last output occurred, and $\{j, k\} \in\{0,1\} \times\{0,1\}$ is the number of customers left behind at servers 1 and 2. Since all the service and interarrival distributions are negative exponential, this process is Markov renewal. The five possible states are $\{1,0,0\},\{2,0,0\},\{2,1,0\},\{1,0,1\}$, and the overflow state, which does not require the index of the last output, $\{*, 1,1\}$. With the states in that order, and using the notation $\lambda+s=a, \lambda+\mu+s=b, \lambda+2 \mu+s=c$, the semi-Markov kernel is then

$$
Q_{s}^{d}=\left(\begin{array}{ccccc}
\frac{\lambda \mu}{2 a b} & \frac{\lambda \mu}{2 a b} & \frac{\lambda^{2} \mu}{a b c} & \frac{\lambda^{2} \mu}{a b c} & \frac{\lambda^{3}}{a b c} \\
\frac{\lambda \mu}{2 a b} & \frac{\lambda \mu}{2 a b} & \frac{\lambda^{2} \mu}{a b c} & \frac{\lambda^{2} \mu}{a b c} s & \frac{\lambda^{3}}{a b c} \\
\frac{\mu}{b} s & 0 & \frac{\lambda \mu}{a b c} & \frac{\lambda \mu}{a b c} & \frac{\lambda^{2}}{b c} \\
0 & \frac{\mu}{b} & \frac{\lambda \mu}{a b c} & \frac{\lambda \mu}{a b c} & \frac{\lambda^{2}}{b c} \\
0 & 0 & \frac{\mu}{b} & \frac{\mu}{b} & \frac{\lambda}{c}
\end{array}\right) .
$$




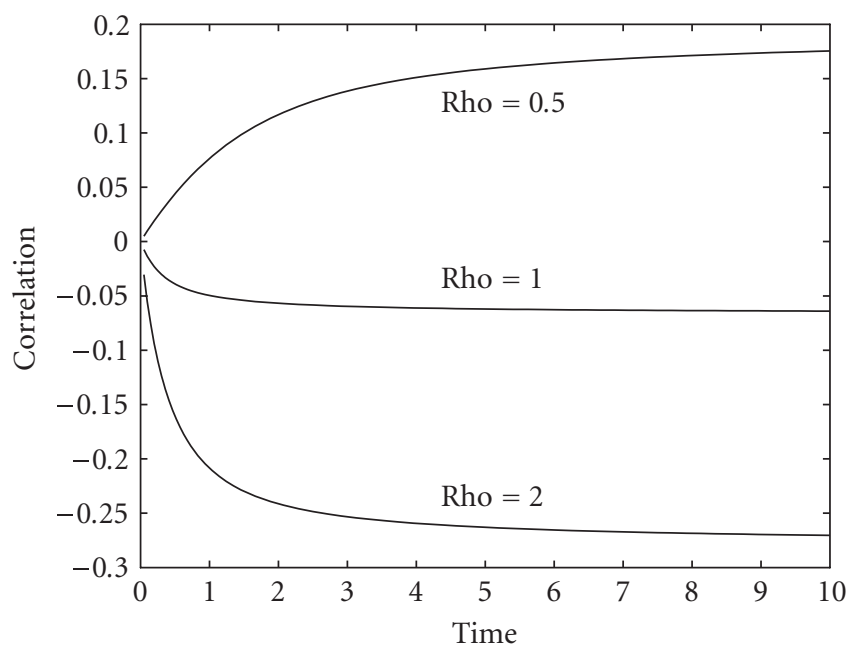

Figure 3.3. M/M/2/0 total output and overflow correlations.

Now that we have two departure streams, there are a number of cross-correlations that could be considered. We give only those between the total output process (i.e., the superposition of the outputs from the two servers) and the overflow process, in Figure 3.3. Thus the results are qualitatively similar to the first system, $\mathrm{M} / \mathrm{M} / 1 / 0$.

3.4. A remark on the equivalent random method. Since this is a simple case of the classic Erlang-B situation, it is also interesting to look at the cross-correlation between the two output streams. Even in the case where the servers are tested in fixed order, these turn out to be very small $(<0.05$ in absolute value. $)$ This may suggest another reason why mean-variance methods like the equivalent random method (see Cooper [3, page 165]) have been found to work so well in telephone networks. If all the output streams, including those from a single set of lines, are nearly uncorrelated, characterising the carried traffic at a subsequent link by only its first two moments is more likely to work.

3.5. Some comments on the results so far. From the results so far, we can form explanations which give some insight into the processes involved. We note that when the traffic intensity is low, the cross-correlations are positive; when the traffic intensity is about 1 , the cross-correlations are very small; and that when the traffic intensity is much greater than 1 , the cross-correlations are strongly negative. Our explanation for this goes as follows. When the traffic intensity is low, the dependence in the departure process is basically being driven by fluctuations in the arrival process. Hence the output and overflow processes tend to move together; when there are an abnormally large number of outputs there are also an abnormally large number of overflows. For high traffic intensity, on the other hand, the server is almost always busy so the output and overflow processes are complements of each other-abnormal excess in one is associated with abnormal paucity in the other, and hence the cross-correlations are negative. 


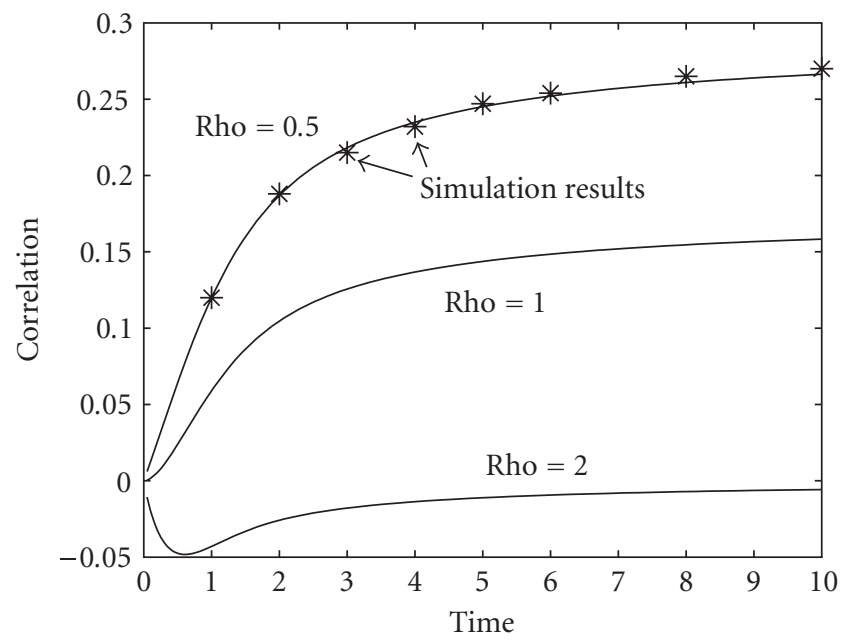

FIgURE 3.4. $\mathrm{M} / \mathrm{E}_{2} / 1 / 0$ output and overflow cross-correlations.

3.6. Nonexponential service time distributions. In general, cross-correlations for these systems are difficult to measure analytically, since the departure process does not have a compact representation as a Markov renewal process. One tractable case is $M / E_{2} / 1 / 0$. The state is the phase of the customer (if any) left behind in service by a departure. Thus state 0 corresponds to an output, while state 1 is an overflow that occurred while the customer in service was in the first phase of service, and state 2 is an overflow that occurred during the second phase of service. The semi-Markov kernel is

$$
Q_{s}^{d}=\left(\begin{array}{ccc}
\frac{\lambda \mu^{2}}{(\lambda+s)(\lambda+\mu+s)^{2}} & \frac{\lambda^{2}}{(\lambda+s)(\lambda+\mu+s)} & \frac{\lambda^{2} \mu}{(\lambda+s)(\lambda+\mu+s)^{2}} \\
\frac{\mu^{2}}{(\lambda+\mu+s)^{2}} & \frac{\lambda}{\lambda+\mu+s} & \frac{\lambda \mu}{(\lambda+\mu+s)^{2}} \\
\frac{\mu}{\lambda+\mu+s} & 0 & \frac{\lambda}{\lambda+\mu+s}
\end{array}\right) .
$$

We again make the mean service time 1, so each stage of the service time distribution has the rate 2, and plot the output and overflow cross-correlations in Figure 3.4, along with some simulation results for the same system (see Section 4).

So the trend of positive cross-correlations at low traffic intensities continues with Erlang service distributions, but the negative cross-correlations at high traffic intensities, although present, are less pronounced, perhaps due to the lower rate of overflows.

\section{Some simulation results}

For Erlang service time distributions with $L>0$ (and for $\mathrm{M} / \mathrm{M} / 1 / \mathrm{L}$ with $L>2$ ), the Markov renewal representation of the departure process is either too complex or has too 
many states to use the analytic approach detailed in Section 2. Simulating such systems is quite easy however. A simulation programme in GPSS/H was written to see if the effects noted previously carry on to systems with more storage. The simulation programme was first verified against the analytic results for $\mathrm{M} / \mathrm{E}_{2} / 1 / 0$ from the previous section. For each time value 100000 pairs of the number of outputs and the number of overflows were recorded, along with the sample cross-correlation calculated from these. The half width of an approximate 95\% confidence interval for these cross-correlations would be $1.96 / \sqrt{ } 100000=0.006$. All of the simulation results (some are plotted on Figure 3.4 in the previous section) are within this distance of the analytic values.

$M / E_{2} / 1 / 2$. We keep the mean service time to be 1 (so each stage of the Erlang distribution has the rate 2 ).

\begin{tabular}{c|r|r|r|r|r|r|r}
\hline Traffic intensity & \multicolumn{1}{|c}{ Time } & \multicolumn{1}{c}{0} & \multicolumn{1}{c}{2} & \multicolumn{1}{c}{4} & \multicolumn{1}{c}{6} & \multicolumn{1}{c}{0} \\
\hline$\rho=0.5$ & & 0 & 0.089 & 0.155 & 0.190 & 0.211 & 0.221 \\
$\rho=1$ & & 0 & -0.014 & 0.025 & 0.050 & 0.063 & 0.069 \\
$\rho=2$ & & 0 & -0.222 & -0.257 & -0.274 & -0.284 & -0.288 \\
\hline
\end{tabular}

Now with some storage, the results have returned to a pattern compatible with our explanation of the effect of traffic intensity, positive correlations at low traffic intensity as both the overflow and output processes move together, and negative correlations at high traffic intensity, where they tend to be the complements of each other.

$D / M / 1 / 2$. The system change we are after here is the reduced variability in the arrival process. If our explanation of the qualitative effect of traffic intensity on the cross-correlations is correct, we might expect that the low traffic intensity effect of positive cross-correlations would be reduced or eliminated, while the high traffic intensity effect of negative crosscorrelations should be at least preserved or possibly enhanced. That is precisely what happens:

\begin{tabular}{c|c|c|c|c|c|c|c}
\hline Traffic intensity & \multicolumn{1}{|c}{ Time } & \multicolumn{1}{c}{0} & \multicolumn{2}{c}{4} & \multicolumn{1}{c}{6} & \multicolumn{1}{c}{8} & 10 \\
\hline$\rho=0.5$ & & 0 & -0.007 & -0.032 & -0.061 & -0.082 & -0.106 \\
$\rho=1$ & & 0 & -0.329 & -0.485 & -0.591 & -0.665 & -0.717 \\
$\rho=2$ & & 0 & -0.705 & -0.833 & -0.885 & -0.911 & -0.928 \\
\hline
\end{tabular}

Two other examples, $\mathrm{E}_{2} / \mathrm{M} / 1 / 0$ and $\mathrm{E}_{2} / \mathrm{E}_{2} / 1 / 0$, support the hypothesis that it is the variability of the arrival process that is responsible for the positive cross-correlations at low traffic intensities.

$E_{2} / M / 1 / 0$.

\begin{tabular}{c|c|c|c|c|c|c|c}
\hline Traffic intensity & \multicolumn{1}{|c}{ Time } & \multicolumn{1}{c}{0} & \multicolumn{2}{c}{4} & \multicolumn{2}{c}{6} & \multicolumn{2}{c}{8} & 10 \\
\hline$\rho=0.5$ & & 0 & -0.018 & -0.054 & -0.071 & -0.080 & -0.089 \\
$\rho=1$ & & 0 & -0.019 & -0.249 & -0.268 & -0.279 & -0.283 \\
$\rho=2$ & & 0 & -0.349 & -0.391 & -0.405 & -0.411 & -0.415 \\
\hline
\end{tabular}


$E_{2} / E_{2} / 1 / 0$

\begin{tabular}{c|r|r|r|r|r|r|r}
\hline Traffic intensity & \multicolumn{1}{|c}{ Time } & \multicolumn{1}{c}{0} & \multicolumn{1}{c}{2} & \multicolumn{1}{c}{4} & \multicolumn{1}{c}{6} \\
\hline$\rho=0.5$ & & 0 & 0.071 & 0.064 & 0.051 & 0.045 & 0.043 \\
$\rho=1$ & & 0 & -0.071 & -0.091 & -0.107 & -0.109 & -0.114 \\
$\rho=2$ & & 0 & -0.215 & -0.238 & -0.245 & -0.250 & -0.254 \\
\hline
\end{tabular}

\section{The effects of these correlations}

5.1. How big are the effects of these correlations? Can we ever get away with treating the overflow and output streams as being independent? For the simplest model, M/M/1/0, it is possible to answer this exactly by considering, as an alternative, a model consisting of two independent $\mathrm{M} / \mathrm{M} / 1 / 0$ systems and combining the outputs from the first system and the overflows from the second system. So this alternative combined process is what would result if we were to treat the overflow and output streams as being independent. As a reference model to measure the effects of this assumption, we compare the blocking probability at a subsequent server with no storage, called server 3, both for the alternative model and the correct (Poisson input) model.

We first need to determine the departure process from the two-independent-systems model. We take the state of the process to be $\{i, j, k\}$, where $i=1$ or 2 is the system from which the last output occurred, and $\{j, k\} \in\{0,1\} \times\{0.1\}$ is the number of customers left behind at servers 1 and 2 . Since all the service and interarrival distributions are negatively exponential, this process is Markov renewal. The eight possible states are outputs from node $1:\{1,0,0\},\{1,0,1\}$, overflows from node $1:\{1,1,0\},\{1,1,1\}$, outputs from node 2 : $\{2,0,0\},\{2,0,1\}$, and overflows from node $2:\{2,1,0\},\{2,1,1\}$. The semi-Markov kernel is then

$$
Q=\left(\begin{array}{cccccccc}
\frac{\lambda_{1} \mu_{1}}{a b} & \lambda_{1} \lambda_{2} \mu_{1} e & \frac{\lambda_{1}^{2}}{a b} & \lambda_{1}^{2} \lambda_{2} e & \frac{\lambda_{2} \mu_{2}}{a c} & \frac{\lambda_{2}^{2}}{a c} & \lambda_{1} \lambda_{2} \mu_{1} e & \lambda_{1} \lambda_{2}^{2} e \\
0 & \frac{\lambda_{1} \mu_{1}}{b d} & 0 & \frac{\lambda_{1}^{2}}{c d} & \frac{\mu_{2}}{c} & \frac{\lambda_{2}}{c} & \frac{\lambda_{1} \mu_{2}}{c d} & \frac{\lambda_{1} \lambda_{2}}{c d} \\
\frac{\mu_{1}}{b} & \frac{\lambda_{2} \mu_{1}}{b d} & \frac{\lambda_{1}}{b} & \frac{\lambda_{2} \lambda_{1}}{b d} & 0 & 0 & \frac{\mu_{2}}{d} & \frac{\lambda_{2}}{d} \\
0 & \frac{\mu_{1}}{d} & 0 & \frac{\lambda_{1}}{d} & 0 & 0 & \frac{\mu_{2}}{d} & \frac{\lambda_{2}}{d} \\
\frac{\lambda_{1} \mu_{1}}{a b} & \lambda_{1} \lambda_{2} \mu_{1} e & \frac{\lambda_{1}^{2}}{a b} & \lambda_{1}^{2} \lambda_{2} e & \frac{\lambda_{2} \mu_{2}}{a c} & \frac{\lambda_{2}^{2}}{a c} & \lambda_{1} \lambda_{2} \mu_{1} e & \lambda_{1} \lambda_{2}^{2} e \\
0 & \frac{\lambda_{1} \mu_{1}}{b d} & 0 & \frac{\lambda_{1}^{2}}{c d} & \frac{\mu_{2}}{c} & \frac{\lambda_{2}}{c} & \frac{\lambda_{1} \mu_{2}}{c d} & \frac{\lambda_{1} \lambda_{2}}{c d} \\
\frac{\mu_{1}}{b} & \frac{\lambda_{2} \mu_{1}}{b d} & \frac{\lambda_{1}}{b} & \frac{\lambda_{2} \lambda_{1}}{b d} & 0 & 0 & \frac{\mu_{2}}{d} & \frac{\lambda_{2}}{d} \\
0 & \frac{\mu_{1}}{d} & 0 & \frac{\lambda_{1}}{d} & 0 & 0 & \frac{\mu_{2}}{d} & \frac{\lambda_{2}}{d}
\end{array}\right) .
$$




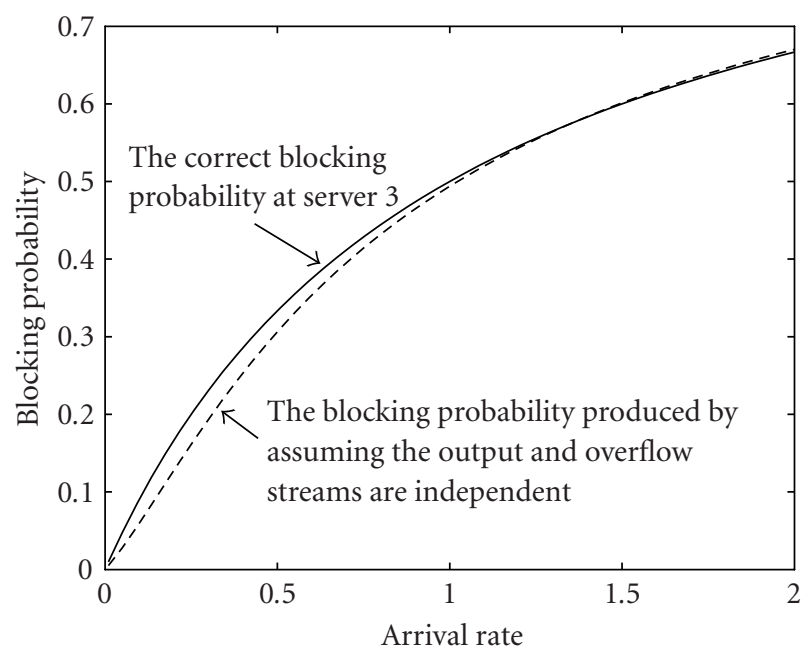

FIGURE 5.1. The effect of treating output and overflow streams as being independent.

Here $a=\lambda_{1}+\lambda_{2}+s, b=\lambda_{1}+\lambda_{2}+\mu_{1}+s, c=\lambda_{1}+\lambda_{2}+\mu_{2}+s, d=\lambda_{1}+\lambda_{2}+\mu_{1}+\mu_{2}+s$, and $e=1 / a b d+1 /$ acd . The input process that we require constitutes entries to states $1,2,7$, and 8 so this is obtained from Q by the usual filtering arguments. That is, if $S=\{1,2,7,8\}$ and $T=\{3,4,5,6\}$, the Laplace-Stieltjes transform of the semi-Markov kernel of the input process is found from: $Q_{i}=Q_{S S}+Q_{S T}\left(I-Q_{T T}\right)^{-1} Q_{T S}$, where $Q_{S S}, Q_{S T}, Q_{T T}$, and $Q_{T S}$ are the corresponding submatrices of $Q$.

Finally when used as input to a subsequent node (server 3 ) with no storage and service rate $\mu_{3}$, the state distribution as seen by an arriving customer has a semi-Markov kernel given by

$$
Q_{3}(s)=\left(\begin{array}{ll}
Q_{i}(s)-Q_{i}\left(s+\mu_{3}\right) & Q_{i}\left(s+\mu_{3}\right) \\
Q_{i}(s)-Q_{i}\left(s+\mu_{3}\right) & Q_{i}\left(s+\mu_{3}\right)
\end{array}\right) .
$$

Since states $5,6,7,8$ of this process are the states in which an overflow at server 3 occurs, the probability of overflow at node 3 is $\pi_{5}+\pi_{6}+\pi_{7}+\pi_{8}$, taken from the steady state distribution of the imbedded Markov chain $Q_{3}(0)$.

If we take $\lambda_{1}=\lambda_{2}$, and all of the service rates to be one, the fraction of customers who overflow from server 3 is simplified to

$$
\pi_{5}+\pi_{6}+\pi_{7}+\pi_{8}=\frac{\lambda\left(4 \lambda^{5}+25 \lambda^{4}+67 \lambda^{3}+88 \lambda^{2}+49 \lambda+60\right)}{\left(4 \lambda^{4}+13 \lambda^{3}+16 \lambda^{2}+9 \lambda+1\right)\left(\lambda^{2}+4 \lambda+6\right)} .
$$

We can compare this with the overflow fraction which would occur using the correctly correlated overflow and output processes. This is easy to calculate as server 3 is actually 


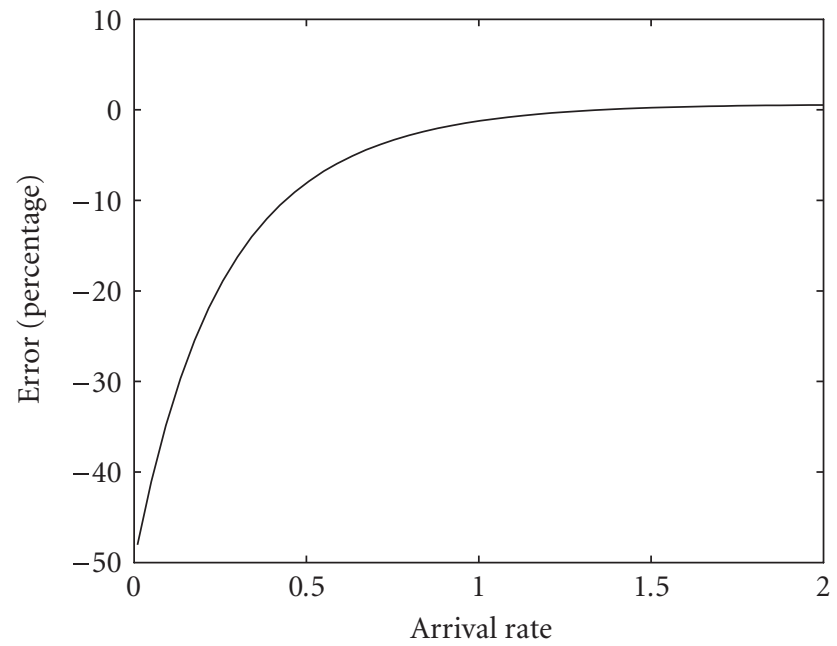

Figure 5.2. Percentage error due to assuming independence.

an $\mathrm{M} / \mathrm{M} / 1 / 0$ system. Plotting the error against traffic intensity (see Figure 5.1 ) shows that while for high traffic intensities the error is small, for intensities less than 1, treating the overflow and output processes as being independent leads to an underestimation of the blocking probability by up to $50 \%$ (see Figure 5.2 ).

5.2. How much extra information can we get from the correlations? In the same case, $\mathrm{M} / \mathrm{M} / 1 / 0$, we can show that there is enough information in the cross-correlations to perfectly reconstruct the departure process. We assume that we know the marginal distributions of the time between outputs and of the time between overflows. However, in addition, we assume we know, or have measured, the cross-covariances (like equation (3.3), but conditioned on the starting state). It is easy to show that we now have enough information in the four equations to solve for, for example, the Markov renewal kernel (3.2). So at least in the class of two-state Markov renewal processes, we would have exactly determined the departure process. Thus at least in this simple case, the addition of this information on cross-correlations is enough to reduce the error to zero.

\section{Conclusions}

We have shown that considerable dependencies, as measured by cross-correlations, can arise in the output and overflow processes from simple queueing models. These can be large, and either positive or negative. Positive cross-correlations are associated with lower traffic intensities, which is also the situation in which ignoring these correlations may produce the largest percentage errors. The variability of the arrival process is an important factor in this. The smallest correlations usually occur at a traffic intensity close to 1 . The qualitative insight these models provide may prove useful in determining a circumstance under which moment or renewal approximations will work well. 


\section{References}

[1] D. C. Boes, "Note on the output of a queuing system," Journal of Applied Probability, vol. 6, no. 2, pp. 459-461, 1969.

[2] D. N. Shanbhag and D. G. Tambouratzis, "Erlang's formula and some results on the departure process for a loss system," Journal of Applied Probability, vol. 10, no. 1, pp. 233-240, 1973.

[3] R. B. Cooper, Introduction to Queueing Theory, Edward Arnold, London, UK, 2nd edition, 1981.

[4] W. Whitt, "Approximating a point process by a renewal process-I: two basic methods," Operations Research, vol. 30, no. 1, pp. 125-147, 1982.

[5] S. L. Albin, "Approximating a point process by a renewal process-II: superposition arrival processes to queues," Operations Research, vol. 32, no. 5, pp. 1133-1162, 1984.

[6] S. L. Albin and S.-R. Kai, "Approximation for the departure process of a queue in a network," Naval Research Logistics Quarterly, vol. 33, no. 1, pp. 129-143, 1986.

[7] M. A. Johnson, "Markov MECO: a simple Markovian model for approximating nonrenewal arrival processes," Communications in Statistics. Stochastic Models, vol. 14, no. 1-2, pp. 419-442, 1998.

[8] I. J. B. F. Adan and V. G. Kulkarni, "Single-server queue with Markov-dependent inter-arrival and service times," Queueing Systems, vol. 45, no. 2, pp. 113-134, 2003.

[9] A. Heindl, "Decomposition of general queueing networks with MMPP inputs and customer losses," Performance Evaluation, vol. 51, no. 2-4, pp. 117-136, 2003, special issue on queueing networks with blocking, Kouvatsos and Balsamo, eds.

[10] R. L. Disney and P. C. Kiessler, Traffic Processes in Queueing Networks: A Markov Renewal Approach, vol. 4 of Johns Hopkins Series in the Mathematical Sciences, Johns Hopkins University Press, Baltimore, Md, USA, 1987.

[11] E. Çinlar, "Markov renewal theory," Advances in Applied Probability, vol. 1, pp. 123-187, 1969.

Don McNickle: Department of Management, University of Canterbury, Christchurch 8140,

New Zealand

Email address: don.mcnickle@canterbury.ac.nz 


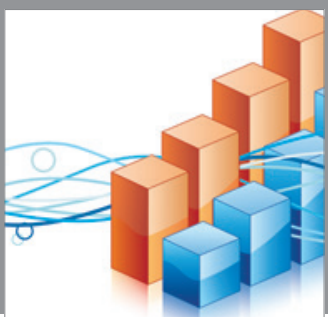

Advances in

Operations Research

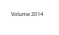

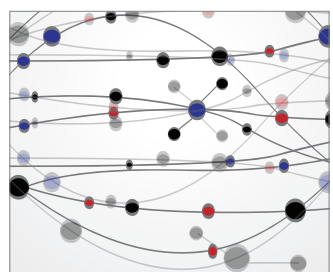

\section{The Scientific} World Journal
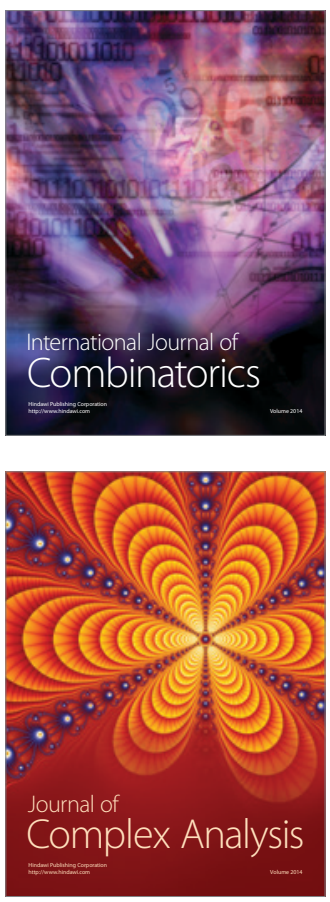

International Journal of

Mathematics and

Mathematical

Sciences
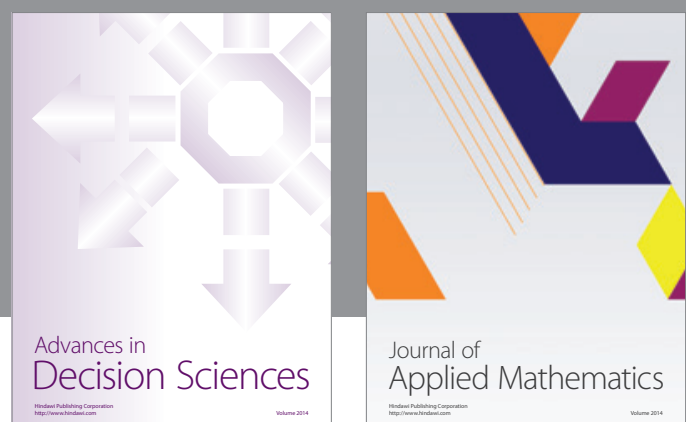

Journal of

Applied Mathematics
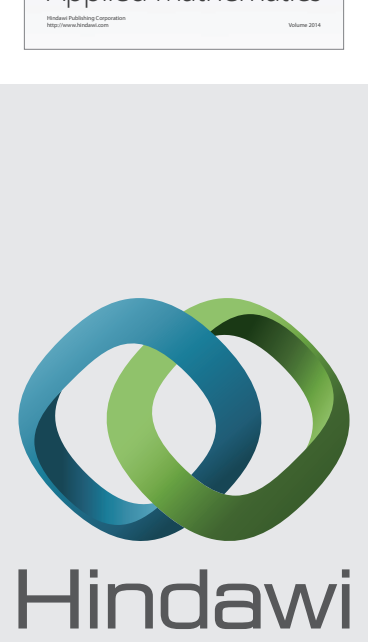

Submit your manuscripts at http://www.hindawi.com
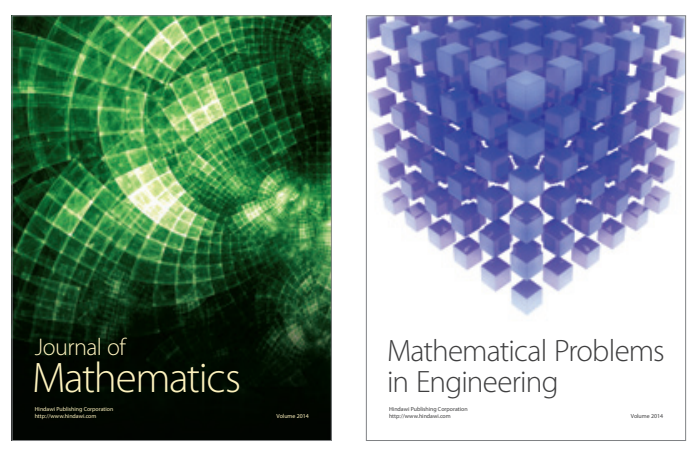

Mathematical Problems in Engineering
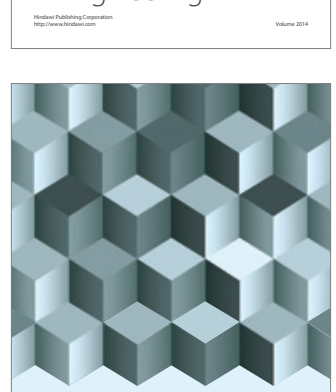

Journal of

Function Spaces
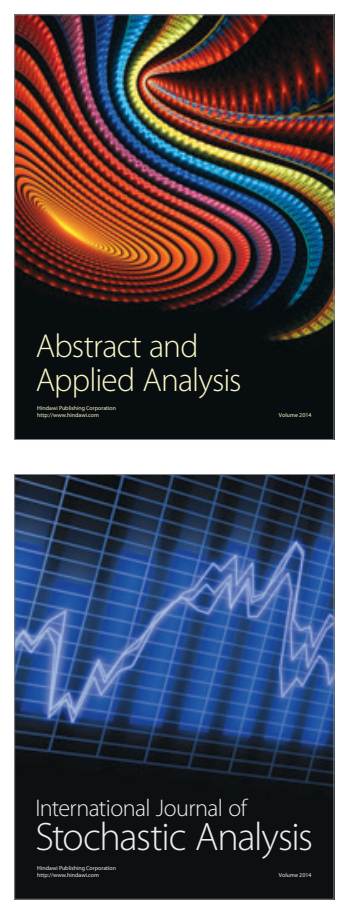

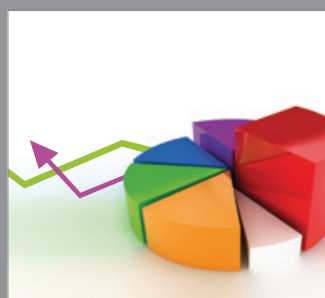

ournal of

Probability and Statistics

Promensencen
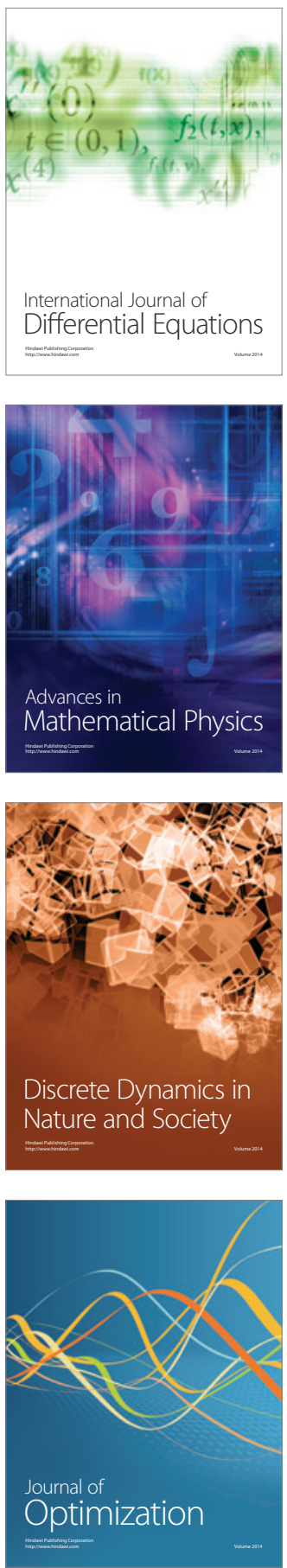